$\sim 16 \mathrm{~mW} \mathrm{~m}^{-2}$, or less than half of that expected in the absence of geochemical differentiation ${ }^{12}$. The surface temperature gradient depends on the integrated crustal radioactivity as well as the mantle heat flux, and may vary from $6 \mathrm{~K} \mathrm{~km}^{-1}$ (where the crustal contribution is not important) to more than $20 \mathrm{~K} \mathrm{~km}^{-1}$ (where it dominates). If the surface temperature is $200 \mathrm{~K}$, the base of the permafrost is at a depth of between 11 and $3 \mathrm{~km}$. Low geothermal gradients early in martian history are also suggested by geophysical estimates of the effective elastic thickness of more than $100 \mathrm{~km}$ beneath the Tharsis volcanoes ${ }^{21}$.

The large volumes of flood water that Baker ${ }^{2}$ and Marsursky et al. ${ }^{16}$ propose to account for the observed landforms must have been released suddenly. The melting process modelled in Fig. 3 generates water far too slowly to maintain flow rates of $10^{7} \mathrm{~m}^{3} \mathrm{~s}-1$. The melt water must therefore first collect, either above or below the surface, before being suddenly released. Valles Marineris contains a number of closed depressions, some of which are dammed by large landslides. Such structures could collect water released from the subsurface, and are likely to undergo catastrophic failure, as they do on Earth.

The mass of water generated by the model in Fig. 3 is comparable to the total mass of the martian atmosphere. Spreading such a layer of water over a large part of Chryse Planitia might therefore have affected the surface temperature and humidity of the entire planet ${ }^{22}$. Most craters in Chryse Planitia show evidence of remobilization of ground ice $^{23}$. This discussion shows that there are no obvious problems with the proposed dyke model, which can account for graben such as Valles Marineris and which can produce the necessary volumes of water for the martian floods. If the above arguments are correct, only a few relatively minor structures on Mars, such as Thaumasia graben ${ }^{1}$, have a purely tectonic origin.

Received 28 January; accepted 19 October 1998

1. Plescia, J. B. \& Saunders, R. S. Tectonic history of the Tharsis Region, Mars. J. Geophys. Res. 87, 9775 9791 (1982).

2. Baker, V. R. The Channels of Mars (Univ. Texas Press, Austin, 1982).

3. Fahrig, W. F. in Mafic Dyke Swarms (eds Halls, H. C. \& Fahrig, W. F.) 331-348 (Spec. Pap. 34, Geol Assoc. Canada, St John's, Newfoundland, 1987).

4. Fahrig, W. F. \& West, T. D. Diabase dyke swarms of the Canadian Shield. (Map 1627A, Geol. Surv. Canada, Ottawa, 1986)

5. Mège, D. \& Masson, P. A plume tectonics model for the Tharsis province, Mars. Planet. Space Sci. 44, 1499-1546 (1996).

6. Basaltic Volcanism Study Project Basaltic Volcanism on Terrestrial Planets (Pergamon, New York, 1983).

7. Ernst, R. E., Head, J. W., Parfitt, E. A., Grosfils, E. \& Wilson, L. Giant radiating dyke swarms on Earth and Venus. Earth Sci. Rev. 39, 1-58 (1995).

8. Sigurdsson, O. Surface deformation of the Krafla fissure swarm in two rifting events. J. Geophys. 47, 154-159 (1980)

9. McKenzie, D., McKenzie, J. M. \& Saunders, R. S. Dike emplacement on Venus and on Earth. J. Geophys. Res. 97, 15977-15990 (1992).

10. Schultz, R. A. Displacement-length scaling for terrestrial and Martian faults: Implications for Valle Marineris and shallow planetary grabens. J. Geophys. Res. 102, 12009-12015 (1997).

11. White, R. S. \& McKenzie, D. Mantle plumes and flood basalts. J. Geophys. Res. 100, 17543-17585 (1995)

12. Schubert, G., Solomon, S. C., Turcotte, D. L., Drake, M. J. \& Sleep, N. H. in Mars (eds Kieffer, H. H., Jakosky, B. M., Snyder, C. W. \& Matthews, M. S.) 147-183 (Univ. Arizona Press, Tucson, 1992).

13. Worst, B. G. The Great Dyke of Southern Rhodesia. S. Rhodesia Geol. Surv. Bull. 47, (1960).

14. Zimbelman, J. R., Craddock, R. A., Greeley, R. \& Kuzmin, R. O. Volatile history of Mangala Valles, Mars. J. Geophys. Res. 97, 18309-18317 (1992).

15. Lucchita, B. K. et al. in Mars (eds Kieffer, H. H., Jakosky, B. M., Snyder, C. W. \& Matthews, M. S.) $453-$ 492 (Univ. Arizona Press, Tucson, 1992).

16. Masursky, H., Boyce, J. M., Dial, A. L., Schaber, G. G. \& Strobell, M. E. Classification and time of formation of Martian channels based on Viking data. J. Geophys. Res. 82, 4016-4038 (1977).

17. Squyres, S. W., Wilhelms, D. E. \& Moosman, A. C. Large-scale volcano-ground ice interaction on Mars. Icarus 70, 385-408 (1987).

18. Squyres, S. W., Clifford, S. M., Kuzmin, R. O., Zimbelman, J. R. \& Costard, F. M. in Mars (eds Kieffer, H. H., Jakosky, B. M., Snyder, C. W. \& Matthews, M. S.) 523-556 (Univ. Arizona Press, Tucson, 1992)

19. Borg, L. E., Nyquist, L. E., Taylor, L. A., Wiesmann, H. \& Shih, C.-Y. Constraints on Martian differentiation processes from Rb-Sr \& Sm-Nd isotopic analyses of the basaltic Shergottite QUE94201. Geochem. Cosmochim. Acta 61, 4915-4931 (1997).

20. Blichert-Toft, J. et al. Lu-Hf isotopic compositions of SNC meteorites: Implications of Martian mantle evolution. Mineral. Mag. A 62, 168-169 (1998).

21. Solomon, S. C. \& Head, J. W. Heterogeneities in the thickness of the elastic lithosphere on Mars: Constraints on heat flow and internal dynamics. J. Geophys. Res. 95, 11073-11083 (1990).

22. Baker, V. R. et al. Ancient oceans, ice sheets and the hydrological cycle on Mars. Nature 352, 589-594 (1991).

23. Carr, M. H. et al. Martian impact craters and emplacement of ejecta by surface flow. J. Geophys. Res. 82, 4055-4065 (1977).

Acknowledgements. We thank J. McKenzie for his help, and the Royal Society, Magdalene College, Cambridge, and NERC for support.

Correspondence and requests for materials should be addressed to F.N. (e-mail: nimmo@esc.cam.ac.uk).

\section{Controlling the shape of a quantum wavefunction}

\section{T. C. Weinacht, J. Ahn \& P. H. Bucksbaum}

Department of Physics, University of Michigan, Ann Arbor, Michigan 48109-1120, USA

The ability to control the shape and motion of quantum states ${ }^{1,2}$ may lead to methods for bond-selective chemistry and novel quantum technologies, such as quantum computing. The classical coherence of laser light has been used to guide quantum systems into desired target states through interfering pathways ${ }^{3-5}$. These experiments used the control of target properties-such as fluorescence from a dye solution ${ }^{6}$, the current in a semiconductor $^{7,8}$ or the dissociation fraction of an excited molecule ${ }^{9}-$ to infer control over the quantum state. Here we report a direct approach to coherent quantum control that allows us to actively manipulate the shape of an atomic electron's radial wavefunction. We use a computer-controlled laser to excite a coherent state in atomic caesium. The shape of the wavefunction is then measured ${ }^{10}$ and the information fed back into the laser control system, which reprograms the optical field. The process is iterated until the measured shape of the wavefunction matches that of a target wavepacket, established at the start of the experiment. We find that, using a variation of quantum holography ${ }^{11}$ to reconstruct the measured wavefunction, the quantum state can be reshaped to match the target within two iterations of the feedback loop.

As in classical holography, quantum holography relies on interference between two waves, which can be labelled as 'object' and 'reference'. Whereas classical holography relies on classical light waves diffracting from scattering media, in quantum holography the object and reference are combined in a single coherently prepared quantum state. This state is a superposition of a shaped wavepacket (the 'object') and a reference wavepacket (the 'signal'). The object and reference packets are created by two specially shaped coherent laser pulses with a time delay, $\tau$, between them. The interference between the object and the reference modulates the spectrum of the wavefunction. A careful measurement of this interference and how it changes with the time delay $\tau$ can be used to reconstruct the object wavefunction ${ }^{10}$. This is similar to the optical technique known as spectral interferometry, which is used to determine the phase and amplitude of a classical light pulse ${ }^{12,13}$.

The essential feature in our application of quantum holography is the ability to measure independently the amplitude of each state in the total wavepacket. This is accomplished with state selective field ionization (SSFI $)^{14}$. SSFI allows us to project the total wavefunction onto the eigenstates of the atomic hamiltonian and directly measure the amplitude of each projection. Eigenstate signals indicate the amplitude of each state in the wavepacket. The correlations in fluctuations of pairs of states indicate the relative phase difference between two states in the object wavepacket and their corresponding states in the reference packet ${ }^{15,16}$. The measured phase and amplitude of each state can be used to reconstruct the object wavepacket as a coherent superposition of eigenstates ${ }^{17-20}$. In the work reported here, this measurement was used as feedback to reshape the optical excitation pulse and thereby reshape the wavepacket.

Both the signal and reference wavepackets were created by irradiating an effusive beam of prepared caesium atoms with two ultrafast, broadband light pulses from an amplified titanium sapphire laser system. The atoms were prepared in the $7 s$ state from the $6 s$ ground state via a two-photon transition driven by an intense nanosecond dye-laser pulse with a wavelength of $1.08 \mu \mathrm{m}$. The ultrafast pulses excited a small portion of the electronic wavefunc- 
tion (consistent with first-order perturbation theory) to the $n p$ Rydberg series. Both ultrafast pulses originated from the same Kerrlens mode-locked (KLM) titanium sapphire laser. The output of the KLM laser was sent through a dispersive grating-pulse expander ${ }^{21}$ and amplified in a $10-\mathrm{Hz}$ regenerative amplifier. The light from the amplifier was split to form two pulses. One of these pulses was directed into an optical pulse shaper, which consisted of a spectrally resolved Bragg deflector at the Fourier plane of a zero-dispersion pulse stretcher ${ }^{22}$. The acoustic waveform on the Bragg deflector was programmable, with independent control of phase and amplitude. This allowed us to reshape the optical pulse to any field consistent with the available optical and acoustic bandwidth ${ }^{22}$. To compensate for the inefficiency of the pulse shaper $(<10 \%)$, the shaped pulse was reamplified in a low-gain, linear multipass amplifier and then recompressed in a parallel grating compressor. The second pulse from the regenerative amplifier was not shaped or reamplified but simply recompressed in a second parallel grating compressor to yield a 150-fs nearly transform-limited pulse. The two pulses were recombined on an optical beam splitter and sent into the vacuum chamber containing the atomic beam.

Our measurements were possible despite the fact that the shaped and reference pulses were not phase-locked. The absence of phaselocking meant that the first-order coherence in the field formed by the two pulses averaged to zero after multiple laser shots. We therefore looked at the second-order coherence of the wavepacket consisting of correlations in the fluctuations of the eigenstate populations resulting from interference between the two excitations. This is essentially a Hanbury-Brown and Twiss measurement applied to atoms ${ }^{16}$. These authors used correlations in the temporal fluctuations of starlight observed by two spatially separate telescopes to measure the size of a star. In the case of atoms, we used spectrally resolved fluctuations of electronic probability to measure the phase of the electronic wavefunction.

The total wavepacket created by the coherent sum of the signal and reference for each laser shot can be written as:

$$
\Psi_{\text {total }}(t=\tau)=\sum_{n=24}^{31}\left(a_{n} \mathrm{e}^{-\mathrm{i} \omega_{n} \tau}+b_{n} \mathrm{e}^{-i \omega_{\mathrm{gs}} \tau}\right) \psi_{n}
$$

where $a_{n}=\left|a_{n}\right| \mathrm{e}^{i \delta_{n}}$. The complex coefficients $a_{n}$ were determined by the shaped laser pulse and the real $b_{n}$ s were determined by the spectrum of the transform-limited reference pulse. The $\psi_{n}$ represent the atomic Rydberg $n p$ eigenstates with principal quantum number $n$ ranging from 24 to 31 . The phase acquired by each state during the time delay $\tau$ between the two pulses $\left(\omega_{n} \tau\right)$ depended on the energy of each state. The phase acquired by the ground state during this same time delay was $\left(\omega_{\mathrm{gs}} \tau\right)$.

The population of each eigenstate in the wavepacket (the square modulus of the projection of the total wavefunction onto the eigenstate) was measured by SSFI. This consisted of exposing the Rydberg atoms in the beam to a linearly increasing electric field as a function of time. Each eigenstate in the wavepacket had a different field ionization threshold, and hence ionized at a different time. The ionization signal as a function of time, as measured by an electron multiplier, displayed a series of peaks whose integrals were proportional to the population of each eigenstate. The population of each eigenstate as measured by SSFI was:

$$
P_{n}=\left|a_{n}\right|^{2}+\left|b_{n}\right|^{2}+2\left|a_{n}\right|\left|b_{n}\right| \cos \left(\left(\omega_{n}-\omega_{\mathrm{gs}}\right) \tau-\delta_{n}\right)
$$

In the absence of a reference pulse, the coefficients $P_{n}$ still yield the $\left|a_{n}\right|$ directly, but without the second wavepacket the $P_{n}$ cannot give any information about the phase of these complex coefficients.

The destruction of first-order coherence over several laser pulses is due to small random variations in $\tau$ of the order of an optical period. These fluctuations can be caused by slight air currents and table vibrations. In our experiment, there was also a random phase introduced between signal and reference pulses by the carrier
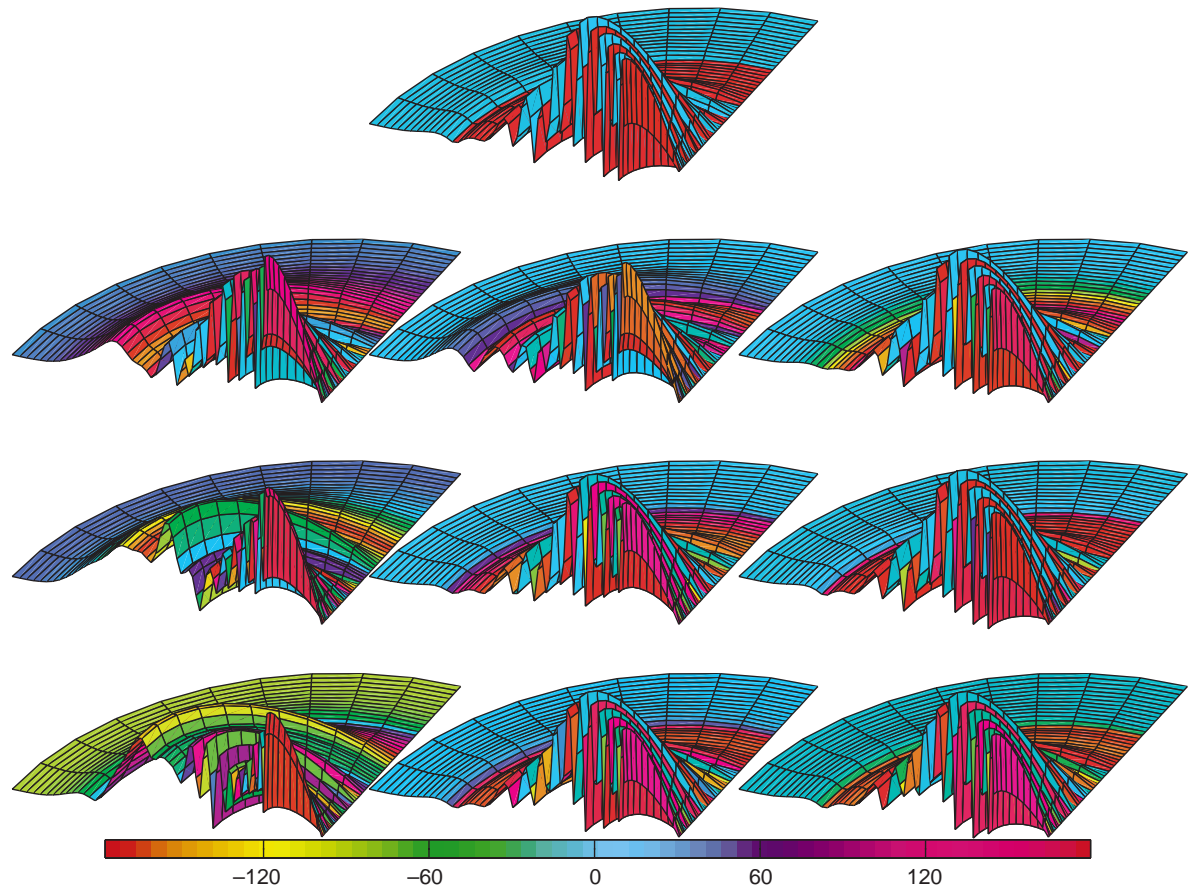

Figure 1 Atomic Rydberg wavefunctions reconstructed from measured projections. The top panel shows the target wavefunction which is the only wavefunction that was not measured. For the other panels, new rows show wavefunctions measured at new time delays, and columns show successive iterations in the feedback loop. The axes are not shown explicitly in order to conserve space but they are as follows: the vertical axis shows the amplitude of

the wavefunction in atomic units ( 1 a.u. $=0.529 \AA$ ), colour denotes phase, the axis to the right is the $z$ axis and the other is the $x$ axis. The range of the $x$ and $z$ axes is 1,800 a.u. and the vertical axis range is $1.5 \times 10^{-3}$ a.u. The phase shown ranges from -180 degrees to 180 degrees and is illustrated by the colour bars shown at the bottom of the figure (in degrees). The excitation laser was polarized along the $x$-axis. 
frequency of the acoustic wave in the Bragg deflector, which was not phase-locked to the output of the KLM laser. Over several laser shots, the cosine term in the $P_{n}$ averages to zero and any interference yielding the relative phase between states $\delta_{n m}$ washes out. In general, measurements of first-order coherence oscillate at the laser frequency $\omega_{\mathrm{L}}=\omega_{n}-\omega_{\mathrm{gs}}$ and therefore vanish in ensemble averages with variations of delay on the order of $2 \pi / \omega_{\mathrm{L}}$ :

$$
\left\langle\left\langle\Psi_{\text {signal }} \mid \Psi_{\text {reference }}\right\rangle\right\rangle \propto\left\langle\sum_{n=24}^{31} \cos \left(\left(\omega_{n}-\omega_{\mathrm{gs}}\right) \tau-\delta_{n}\right)\right\rangle=0
$$

Measurements involving second-order coherence contain oscillations at the Kepler frequency (frequency differences in the laser bandwidth), and therefore retain the desired phase information while averaging over several laser shots for these same temporal variations:

$\left\langle\left\langle\Psi_{\text {signal }} \mid \Psi_{\text {reference }}\right\rangle\left\langle\Psi_{\text {signal }} \mid \Psi_{\text {reference }}\right\rangle\right\rangle \propto\left\langle\sum_{n, m} \cos \left(\left(\omega_{n}-\omega_{m}\right) \tau-\delta_{n m}\right)\right\rangle \neq 0$

We made use of the second-order coherence of the wavepackets by constructing the normalized correlation coefficient between pairs of eigenstate populations:

$$
r_{n m}=\frac{\left.\left\langle P_{n} P_{m}\right\rangle-\left\langle P_{n}\right\rangle P_{m}\right\rangle}{\left(\Delta P_{n}\right)\left(\Delta P_{m}\right)}=\cos \left(\left(\omega_{n}-\omega_{m}\right) \tau-\delta_{n m}\right)
$$

In this expression, the factors $\Delta P_{n}$ are the standard deviations in the populations $P_{n}$.

This result also permitted us to measure the degree of coherence with the following procedure: we programmed the Bragg deflector to step each state in the wavepacket through a series of phases with respect to each of the other states in the wavepacket. The phase difference between any two states in the wavepacket was determined by fitting $r_{n m}$ to a cosine curve. Decoherence of the shaped wavepacket caused a decrease in the amplitude of the measured cosine curve. Using this method, we were able to detect coherence in the shaped wavepacket lasting beyond $1 \mathrm{~ns}$ for atoms at a temperature of $400 \mathrm{~K}$.

Knowing the amplitude and relative phase of each state in the wavepacket, the electronic wavefunction could be completely reconstructed ${ }^{10}$. Once reconstructed, the difference between the measured wavefunction and the desired target state was computed and used to reprogam the phase of each state via the Bragg deflector. Having the full reconstruction information obviated the need for a sophisticated algorithm to implement feedback, and resulted in very rapid convergence to any target that we chose. In our simple feedback algorithm, the relative phase of each projection was adjusted by an amount equal to the difference between the measured phase and the target phase. For any given delay $\tau$, the algorithm was able to control the shaped wavefunction to match the target within two iterations of the feedback loop. Nonlinearities in the Bragg deflector response and other technical control problems were automatically corrected in the feedback loop.

The system was capable of adapting to changing initial conditions and maintaining a fixed output (the target wavefunction) with a changing input. This was demonstrated by changing the measurement time delay $\tau$ after the algorithm converged. The algorithm adapted to the new launch time and compensated for the extra dispersion introduced by the new delay such that the wavepacket matched the target at the new measurement time. The measurement delay was altered several times, and each time the algorithm converged quickly. Figure 1 shows the shaped wavepacket following each iteration. The top frame shows the target wavefunction. All other frames show measured wavefunctions at the time the reference pulse was incident on the atoms. Each row starts with a different delay setting, and each new column shows the next iteration in the feedback loop.
The rapid convergence of this experiment is due partly to the fact that the excitation of the shaped wave packet was linear in light fluence, that is, the excitation was in the weak-field limit. This is not a requirement for the measurement technique, nor is it a limitation on the application of wavefunction reconstruction to coherent control. As long as the reference pulse excites a wavepacket in the weak field limit, the phase and amplitude of any wavepacket interfering with the reference can be measured. We are currently using this technique to measure wavepackets created by half-cycle pulses $^{23}$, which interact with the atom in a highly non-perturbative fashion.

If the initial wavepacket is created in the strong-field regime, then convergence will not be as rapid as in this experiment and a more sophisticated algorithm will be required to implement control with feedback. However, knowledge of the full wavefunction can be used to direct the search for the optimal pulse shape and minimize the amount of phase space that has to be explored. Furthermore, the reconstruction provides a direct comparison between the results of the optimal pulse shape and the target.

We have demonstrated active control of a Rydberg atomic electron via holographic reconstruction. The reconstructed wavefunction guided control of its shape. Direct applications of this technique include using shaped Rydberg atoms as transient nanostructures for time-gated diffraction of X-rays ${ }^{24}$ and as storage and computation modules for quantum information processing and computing. Along more general lines, this experiment serves as an illustration of the utility of quantum state reconstruction as another tool for achieving coherent control via feedback.

Received 30 July; accepted 20 October 1998

1. Warren, W. S., Rabitz, H. \& Dahleh, M. Coherent control of quantum dynamics: the dream is alive. Science 259, 1581-1589 (1993).

2. Gordon, R. J. \& Rice, S. A. Active control of the dynamics of atoms and molecules. Annu. Rev. Phys. Chem. 48, 601-641 (1997).

3. Tannor, D. J. \& Rice, S. A. Coherent pulse sequence control of product formation in chemicalreactions. Adv. Chem. Phys. 70, 441-523 (1998).

4. Shapiro, M. \& Brumer, P. Coherent and incoherent laser control of photochemical-reactions. Int. Rev. Phys. Chem. 13, 187-229 (1994).

5. Judson, R. S. \& Rabitz, H. Teaching lasers to control molecules. Phys. Rev. Lett. 68, 1500-1503 (1992). 6. Bardeen, C. J. et al. Feedback quantum control of molecular electronic population transfer. Chem. Phys. Lett. 280, 151-158 (1997).

7. Dupont, E., Corkum, P. B., Liu, H. C., Buchanan, M. \& Wasilewski, Z. R. Phase-controlled currents in semiconductors. Phys. Rev. Lett. 74, 3596-2599 (1995).

8. Hache, A. et al. Observation of coherently controlled photocurrent in unbiased, bulk GaAs. Phys. Rev. Lett. 78, 306-309 (1997).

9. Kleiman, V., Zhu, L., Li, X. \& Gordon, R. J. Coherent phase control of the photoionization of $\mathrm{H}_{2} \mathrm{~S}$. J. Chem. Phys. 102, 5863-5866 (1995).

10. Weinacht, T. C., Ahn, J. \& Bucksbaum, P. H. Measurement of the amplitude and phase of a sculpted Rydberg wave packet. Phys. Rev. Lett. 80, 5508-5511 (1998).

11. Leichtle, C., Schleich, W. P., Averbukh, I. S. \& Shapiro, M. Quantum state holography. Phys. Rev. Lett. 80, 1418-1421 (1998)

12. Xin, C. \& Yeazell, J. A. Analytical wave-packet design scheme: control of dynamics and creation of exotic wave packets. Phys. Rev. A 56, R2274-R2277 (1997).

13. Fittinghoff, D. N. et al. Measurement of the intensity and phase of ultraweak, ultrashort laser pulses. Opt. Lett. 21, 884-886 (1996).

14. Gallagher, T. F., Humphrey, L. M., Cooke, W. E., Hill, R. M. \& Edelstein, S. A. Field ionization of highly excited states of sodium. Phys. Rev. A 16, 1098-1108 (1977).

15. Kinrot, O., Averbukh, I. S. \& Prior, Y. Measuring coherence while observing noise. Phys. Rev. Lett. 75, 3822-3825 (1995).

16. Hanbury-Brown, R. \& Twiss, R. Q. Correlation between photons in two coherent beams of light. Nature 175, 27-29 (1956).

17. Schleich, W. \& Raymer, M. Quantum state preparation and measurement. J. Mod. Opt. 44, 2021-2022 (1997).

18. Freyberger, M., Bardroff, P., Leichtle, C., Schrade, G. \& Schleich, W. The art of measuring quantum states. Phys. World 10, 41-45 (1997).

19. Leibfried, D., Pfau, T. \& Monroe, C. Shadows and mirrors: reconstructing quantum states of atom motion. Phys. Today 51, 22-28 (1998).

20. Leonhardt, U. Measuring the Quantum State of Light (Cambridge Univ. Press, 1997).

21. Strickland, D. \& Mourou, G. Compression of amplified chirped optical pulses. Opt. Commun. 55, 447-449 (1985).

22. Tull, J. X., Dugan, M. A. \& Warren, W. S. High resolution, ultrafast laser pulse shaping and its applications. Adv. Opt. Magn. Reson. 20, 1-51 (1996).

23. Raman, C., Conover, C. W. S., Sukenik, C. I. \& Bucksbaum, P. H. Ionization of Rydberg wave packets by subpicosecond, half-cycle electromagnetic pulses. Phys. Rev. Lett. 76, 2436-2439 (1996).

24. Krause, J. L., Schafer, K. J., Ben-Nun, M. \& Wilson, K. R. Creating and detecting shaped Rydberg wave packets. Phys. Rev. Lett. 79, 4978-4981 (1997).

Acknowledgements. We thank D. Tannor, J. Krause and J. Cohen for discussions. This work was supported by the NSF.

Correspondence and requests for materials should be addressed to T.C.W. (e-mail: tweinach@umich. edu). 